\title{
Bilateral Teleoperation of Groups of UAVs with Decentralized Connectivity Maintenance
}

\author{
Paolo Robuffo Giordano*, Antonio Franchi*, Cristian Secchi ${ }^{\dagger}$, and Heinrich H. Bülthoff* ${ }^{*}$ \\ *Max Planck Institute for Biological Cybernetics, Tübingen, Germany. \\ Email: \{paolo.robuffo-giordano,antonio.franchi\} @ tuebingen.mpg.de \\ ${ }^{\dagger}$ Department of Science and Methods of Engineering, University of Modena and Reggio Emilia, Italy. \\ Email: cristian.secchi@unimore.it \\ ${ }^{\ddagger}$ Department of Brain and Cognitive Engineering, Korea University. Email: hhb@tuebingen.mpg.de
}

\begin{abstract}
In this paper, we present a decentralized passivitybased control strategy for the bilateral teleoperation of a group of Unmanned Aerial Vehicles (UAVs). The human operator at the master side can command the group motion and receive suitable force cues informative about the remote environment. By properly controlling the energy exchanged within the slave side (the UAV group), we guarantee that the connectivity of the group is preserved and we prevent inter-agent and obstacle collisions. At the same time, we allow the behavior of the UAVs to be as flexible as possible with arbitrary split and join maneuvers. The results of the paper are validated by means of human/hardwarein-the-loop (HHIL) simulations.
\end{abstract}

\section{INTRODUCTION}

For several applications like data collection, surveillance, search and rescue and exploration of wide areas, the use of a group of simple robots rather than a single complex robot has proven to be very effective, and the problem of coordinating a group of agents has received a lot of attention by the robotics community (see [8] for a survey). Nevertheless, when the tasks to be executed involve complex activities (e.g., navigation in a very cluttered, possibly unknown, environment), complete autonomy is still far to be reached and human's intervention/assistance is necessary. In this context, teleoperation systems, where an operator drives a remote robot through a local interface, allow to exploit human's intelligence to solve tasks too complex for the skills of nowadays robots. In particular, it has been proven that the use of bilateral teleoperation systems, where a force information is fed back to the user, guarantees superior performance with respect to unilateral teleoperation where no feedback is present [4].

A lot of interest is arising in the robotics community in single mobile robot teleoperation, see, for instance, [3, 11, 15] for the case of a flying robot (UAV). However, the bilateral control of multiple slaves through a single master imposes challenges that are absent in the traditional single-master/single-slave systems. For instance, it is not clear what is the best way to dispatch the actions of the master to the slaves and what kind of force information feed back to the master side. In [14], a multi-master/multi-slave teleoperation system with no delay is developed and a centralized strategy for controlling the cooperative behavior of the robots is proposed. In [11], a centralized bilateral control strategy that allows to coordinate the motion between the master and the slaves under arbitrary time delay is proposed. In our view, a main limitation of this and similar approaches lies in the rigidity of the group of teleoperated robots which is not allowed, for example, to actively reshape the formation by means of autonomous split and join maneuvers. Furthermore, because of their higher fault tolerance and lower communication demand, decentralized solutions should be preferred in multi-robot control (see, e.g., [7, 8]).

In order to address these issues, we proposed in a recent work [2] a general framework for establishing a bilateral teleoperation system to control a remote group of mobile robots in a decentralized and flexible way. This was achieved by only requiring 1-hop information within the group, and a single communication link between the human operator and one of the robots (which was then referred to as the 'unique' leader). The operator was able to control the motion of the overall group by acting on the leader, and could feel force cues informative about the presence of obstacles and number of agents at the remote site. However, we did not explicitly consider any particular strategy for ensuring connectivity of the group during motion: a sub-group could still potentially detach from the component connected to the leader - a features not desirable in all tasks. Goal of the present paper is then to extend the approach of [2] to also enforce decentralized connectivity maintenance of the group at all times.

In literature two classes of decentralized connectivity maintenance approaches are present: $i$ ) the conservative methods, which aim at preserving the initial graph topology during the task [1, 5, 10], and ii) the flexible approaches, which allow to switch anytime among any of the connected topologies. These usually produce local control actions aimed at keeping (a decentralized estimate of) $\lambda_{2}$, the second smallest eigenvalue of the graph Laplacian, positive over time [16, 17]. This paper can be considered as an extension of the latter approaches. Indeed, our first main contribution is the design of a novel decentralized control action which simultaneously enforces $(i)$ obstacle/inter-robot collision avoidance, and ( $i i)$ flexible connectivity maintenance of the group despite loss of sensing/visibility due to (too large) inter-robot distance and/or occluding obstacles. The second major contribution is the passivity-based design of such a control strategy. This guarantees the passivity of the whole group (i.e. the slave 
side) and allows the implementation of a stable bilateral teleoperation system by properly coupling the group with a passive master device driven by a human operator.

The rest of the paper is organized as follows: in Sect. II the structure of the slave side and of the decentralized control strategy for connectivity maintenance are illustrated. In Sect. IIII the master side and the overall teleoperation system are described. Finally, results of several human/hardware-inthe-loop (HHIL) simulations are reported in Sect. IV] and Sect. $\mathrm{V}$ concludes the paper.

\section{THE SLAVE SIDE}

Following the framework introduced in [2], we consider the slave side as a group of $N$ agents that can be modeled as floating masses in $\mathbb{R}^{3}$, and which are coupled by means of suitable inter-agent forces. Among all the agents, a special one (leader) is also controlled by the master, while the remaining agents (not controlled by the master) are denoted as followers. Exploiting the port-Hamiltonian modeling formalism [13], we model each agent $i$ as an element storing kinetic energy

$$
\left\{\begin{array}{l}
\dot{p}_{i}=F_{i}^{\lambda}+F_{i}^{e}-B_{i} M_{i}^{-1} p_{i} \\
v_{i}=\frac{\partial \mathcal{K}_{i}}{\partial p_{i}}=M_{i}^{-1} p_{i}
\end{array} \quad i=1, \ldots, N\right.
$$

where $p_{i} \in \mathbb{R}^{3}$ and $M_{i} \in \mathbb{R}^{3 \times 3}$ are the momentum and positive definite inertia matrix of agent $i$, respectively, $\mathcal{K}_{i}\left(p_{i}\right)=\frac{1}{2} p_{i}^{T} M_{i}^{-1} p_{i}$ is the kinetic energy stored by the agent during its motion, and $B_{i} \in \mathbb{R}^{3 \times 3}$ is a positive definite matrix representing a velocity damping term (this can be either artificially introduced, or representative of typical phenomena of aerial robots such as wind/atmospehere drag). The force input $F_{i}^{\lambda} \in \mathbb{R}^{3}$ represents the interaction of agent $i$ with the other agents and surrounding environment (obstacles) and $F_{i}^{e} \in \mathbb{R}^{3}$ is an additional external force. Finally, $v_{i} \in \mathbb{R}^{3}$ is the velocity of the agen 1

Two agents are able to communicate, to measure their relative position, and ultimately to interact if and only if they are neighbors according to the following definition:

Definition 1: Agents $i$ and $j$ are neighbors if and only if: i) their relative distance $d_{i j}$ is less than $D \in \mathbb{R}^{+}$(the sensing range) and larger than $d_{\min } \in[0, D)$ (the safety range), ii) their line-of-sight is not occluded by an obstacle, and iii) neither $i$ nor $j$ are closer than $d_{\min }$ to any other agent.

The two latter conditions distinguish our work from most of the past literature on connectivity maintenance and, as will be explained in Sect. III-A, are crucial for embedding both obstacle and inter-agent/collision avoidance within a unified connectivity-preserving action ${ }^{2}$

\footnotetext{
${ }^{1}$ Here we assume that the UAVs are endowed with a low-level controller able to track the dynamical behavior reported in Eq. (1). Similar assumptions have been made in previous related works (e.g., [15]).

${ }^{2}$ Although not commonly considered in the literature, enforcing a minimum inter-agent safe distance $d_{\mathrm{min}}$ is also relevant in many practical circumstances involving local sensing such as, e.g., limited focusing range of on-board cameras, occlusions due to too close agents, or a generic safe distance to prevent dangerous collisions.
}

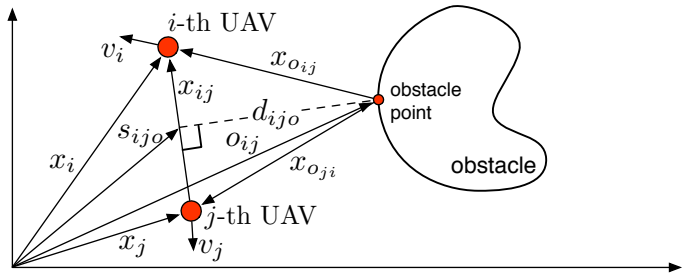

Fig. 1: The agents $i$ and $j$ and their closest obstacle point with the main symbols defined in the paper.

The neighboring relationship defines an undirected graph $\mathcal{G}=(\mathcal{V}, \mathcal{E})$ where the vertices represent the agents and an edge $(i, j)$ represents the presence of a communication/interaction link between agent $i$ and agent $j$. Here, as usual, we denote with $\mathcal{N}_{i}$ the set of neighbors of agent $i$. We now introduce several definitions instrumental for writing in a compact and powerful form the overall slave side.

With reference to Fig. 1. let $x_{i j}=x_{i}-x_{j} \in \mathbb{R}^{3}$ represent the relative position of agent $i$ w.r.t. agent $j$ and

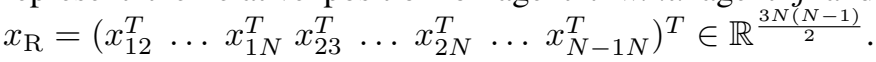
We also assume that an agent can measure the distance from the surrounding obstacles within the range $D$, and we let $\mathcal{O}_{i}$ be a set containing all the obstacle points sensed by agent $i$ plus a fictitious point $o_{\infty}$ at a distance greater than $D$ from any agent (i.e., at infinity). If $j \in \mathcal{N}_{i}$, we let $o_{i j}$ be the closest point in $\mathcal{O}_{i} \cup \mathcal{O}_{j}$ to the segment (line-of-sight) joining agent $i$ and agent $j$. Notice that if $\mathcal{O}_{i} \cup \mathcal{O}_{j}$ does not contain any sensed obstacle point, then we define $o_{i j}=o_{\infty}$. We also set $o_{i j}=o_{\infty}$, by convention, whenever $j \notin \mathcal{N}_{i}$. By letting $x_{o_{i j}}=x_{i}-o_{i j}$ represent the relative position of agent $i$ w.r.t. obstacle point $o_{i j}$, and $x_{o_{i}}=\left(x_{o_{i 1}}^{T} \ldots x_{o_{i k}}^{T} \ldots x_{o_{i N}}^{T}\right)^{T} \in \mathbb{R}^{3(N-1)}, k \neq i$, we can collect all such relative positions for all the $N$ robots into a unique vector $x_{\mathrm{O}}=\left(x_{o_{1}}^{T} \ldots x_{o_{N}}^{T}\right)^{T} \in \mathbb{R}^{3 N(N-1)}$. Analogously to the definition of $x_{\mathrm{O}}$, we also collect all the obstacle point positions $o_{i j}$ into a cumulative vector $o=\left(\ldots, o_{i 1}^{T}, \ldots, o_{i j}^{T} \ldots, o_{i N}^{T} \ldots\right) \in \mathbb{R}^{3 N(N-1)}$, with $i=$ $1, \ldots, N$ and $j \neq i$, and let $v_{o}=\dot{o}$ be the obstacle point velocities seen from the corresponding agent ${ }^{3}$

Finally, we define a suitable scalar lower-bounded potential $V^{\lambda}\left(x_{\mathrm{R}}, x_{\mathrm{O}}\right) \in \mathbb{R}^{+}$whose exact shape will be detailed in Sect. II-A For now, $V^{\lambda}$ is meant to be a generic potential function accounting for connectivity maintenance, inter-agent collision avoidance, and obstacle avoidance for all the $N$ robots in the group. This allows to model the inter-agent and environment interaction as a nonlinear elastic element whose potential energy function is $V^{\lambda}$ and whose gradient $\frac{\partial V^{\lambda}}{\partial\left(x_{R} x_{O}\right)}$ drives the agents toward the desired behavior. We will show that $V^{\lambda}$ is designed in such a way that $x_{i j}$ and $x_{o_{i j}}$ do not contribute to its variation w.r.t. the agent position $x_{i}$ when $j \notin \mathcal{N}_{i}$; thus, the control action implemented on agent $i$ will take the (decentralized) form:

$$
F_{i}^{\lambda}=\sum_{j \in \mathcal{N}_{i}}\left(\frac{\partial V^{\lambda}}{\partial x_{o_{i j}}}+\frac{\partial V^{\lambda}}{\partial x_{i j}}\right) .
$$

\footnotetext{
${ }^{3}$ In fact, even though the obstacles are supposed to be fixed in space, the obstacle points move as the agents move.
} 


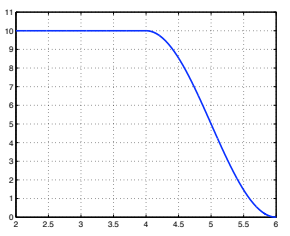

(a)

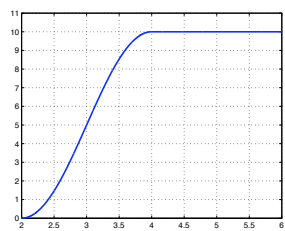

(b)

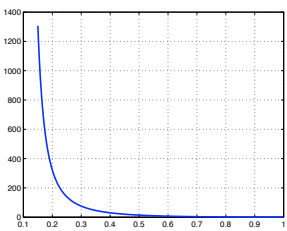

(c)
Fig. 2: The shape of $a_{i j}$ (left), $b_{i j}$ (equiv. $c_{i j}$ ) (center), and $V^{\lambda}\left(\lambda_{2}\right)$ (right).

By defining $p=\left(p_{1}^{T} \ldots p_{N}^{T}\right)^{T} \in \mathbb{R}^{3 N}, B=\operatorname{diag}\left(B_{i}\right)$, and $F^{e}=\left(F_{1}^{e T} \ldots F_{N}^{e T}\right)^{T} \in \mathbb{R}^{3 N}$, and by noting that $\dot{x}_{i j}=v_{i}-$ $v_{j}$, we can then model the overall slave-side as a mechanical system described by:

$\left\{\begin{array}{l}\left(\begin{array}{c}\dot{p} \\ \dot{x}_{R} \\ \dot{x}_{O}\end{array}\right)=\left[\left(\begin{array}{ccc}0 & \mathcal{I} & -\mathbb{I} \\ -\mathcal{I}^{T} & 0 & 0 \\ \mathbb{I}^{T} & 0 & 0\end{array}\right)-\left(\begin{array}{ccc}B & 0 & 0 \\ 0 & 0 & 0 \\ 0 & 0 & 0\end{array}\right)\right] \nabla H+G\left(\begin{array}{c}F^{\mathrm{e}} \\ v_{o}\end{array}\right) \\ \left(\begin{array}{c}v \\ F^{o}\end{array}\right)=G^{T} \nabla H\end{array}\right.$

where

$$
H=\sum_{i=1}^{N} \mathcal{K}_{i}+V^{\lambda} \in \mathbb{R}^{+}
$$

represents the total energy of the system and $\nabla H=$ $\left(\frac{\partial^{T} H}{\partial p} \frac{\partial^{T} H}{\partial x_{\mathrm{R}}} \frac{\partial^{T} H}{\partial x_{O}}\right)^{T}$. Moreover, $\mathcal{I}=\mathcal{I}_{\mathcal{G}} \otimes I_{3}, \mathbb{I}=I_{N} \otimes$ $\mathbf{1}_{N-1}^{T} \otimes I_{3}$, and $G=\left(\begin{array}{ccc}I_{N} \otimes I_{3} & 0 & 0 \\ 0 & 0 & -\mathbb{I}\end{array}\right)^{T}$, with $\mathcal{I}_{\mathcal{G}}$ being the incidence matrix of the graph $\mathcal{G}$ whose edge numbering is induced by the entries of the vector $x_{R}, I_{3}$ and $I_{N}$ being the identity matrices of order 3 and $N$ respectively, $\mathbf{1}_{N-1}$ a column vector of all ones of dimension $N-1,0$ representing a null matrix of proper dimensions, and $\otimes$ denoting the Krönecker product. Finally, $v \in \mathbb{R}^{3 N}$ and $F^{o} \in \mathbb{R}^{3 N(N-1)}$ are the conjugate power variables associated to $F^{e}$ and $v_{o}$, respectively: the system exchanges energy through the port $\left(F^{e}, v\right)$ with the external world, and through the port $\left(v_{o}, F^{o}\right)$ with the obstacles, which are considered as a passive system.

\section{A. The connectivity potential $V^{\lambda}$}

Inspired by [16], we encode the connectivity relationship of Def. 1 into the weights of the links joining neighboring agents. To this end, let $A \in \mathbb{R}^{N \times N}$ be the adjacency matrix associated to the graph $\mathcal{G}$ : each element $A_{i j} \in \mathbb{R}^{+}$, representing the weight of the edge among agents $i$ and $j$, will be designed as a function of $d_{i 1}, \ldots, d_{i N}, d_{j 1}, \ldots, d_{j N}, d_{i j o}$, where $d_{i j}=$ $\left\|x_{i j}\right\|$ is the distance among agents $i$ and $j$, and $d_{i j o}$ the distance between the segment $s_{i j}$ connecting $x_{i}$ with $x_{j}$ (their line-of-sight) and the closest obstacle point $o_{i j}$ (see Fig. 11. The weights $A_{i j}$ are defined as a product of terms which vanish whenever one of the three properties in Def. 1 is not fulfilled (see Fig. 3):

$$
A_{i j}=a_{i j} b_{i j} c_{i} c_{j}, \quad c_{i}=\prod_{k \mid d_{i k} \leq D} c_{i k},
$$

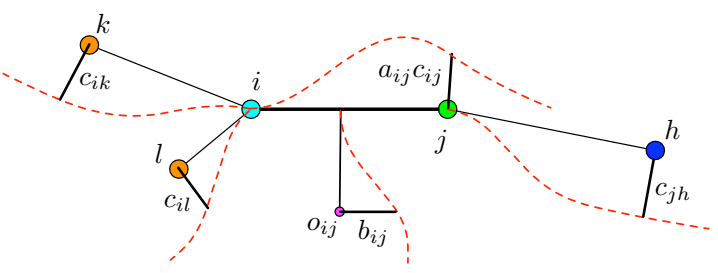

Fig. 3: Graphical representation of the terms contributing to $A_{i j}=$ $a_{i j} b_{i j} c_{i j} c_{i k} c_{i l} c_{j h}$. In this example $k$ and $l$ are the neighbors of $i$, $h$ is the neighbor of $j$, and $o_{i j}$ is the closest obstacle point to the segment joining $i$ and $j$.

with $a_{i j}\left(d_{i j}\right)$ accounting for the constraint on inter-robot maximum sensing range $D, b_{i j}\left(d_{i j o}\right)$ for the constraint on line-ofsight/obstacle distance (and implicitly for obstacle avoidance), and the $c_{i k}\left(d_{i k}\right)$ accounting for the constraint on inter-robot minimum distances (i.e., to avoid inter-agent collisions).

The weights $a_{i j}$ are chosen to stay constant at a maximum value $k_{a}$ for $0 \leq d_{i j} \leq d_{0}$, where $0<d_{0}<D$ is a desired inter-robot distance, and to smoothly vanish (with vanishing derivative) if $d_{i j}=D$. To this end, we chose the following function

$$
a_{i j}\left(d_{i j}\right)=\left\{\begin{array}{cc}
k_{a} & 0 \leq d_{i j} \leq d_{0} \\
\frac{k_{a}}{2}\left(1+\cos \left(\alpha_{a} d_{i j}+\beta_{a}\right)\right) & d_{0}<d_{i j} \leq D \\
0 & d_{i j}>D
\end{array}\right.
$$

with $\alpha_{a}=\frac{\pi}{D-d_{0}}$, and $\beta_{a}=-\alpha_{a} d_{0}$. Figure $2 \mathrm{a}$ shows the shape of $a_{i j}$ for $d_{0}=4, D=6$, and $k_{a}=10$. As for $b_{i j}$, assume a minimum and maximum distance $0 \leq d_{\min }^{o}<d_{\max }^{o} \leq D$ between the segment $s_{i j}$ and $o_{i j}$ are chosen: the weights $b_{i j}$ are defined as

$b_{i j}\left(d_{i j o}\right)=\left\{\begin{array}{cc}0 & d_{o i j} \leq d_{\min }^{o} \\ \frac{k_{b}}{2}\left(1-\cos \left(\alpha_{b} d_{i j o}+\beta_{b}\right)\right) & d_{\min }^{o}<d_{i j o} \leq d_{\max }^{o} \\ k_{b} & d_{i j o}>d_{\max }^{o}\end{array}\right.$

with $\alpha_{b}=\frac{\pi}{d_{\max }^{o}-d_{\min }^{o}}$ and $\beta_{b}=-\alpha_{b} d_{\min }^{o}$. Figure $2 \mathrm{~b}$ shows the shape of $b_{i j}$ for $d_{\min }^{o}=2, d_{\max }^{o}=4$, and $k_{b}=10$. Finally, weights $c_{i k}$ are defined as being constant at a value $k_{c}$ if $d_{i k} \geq d_{0}$, and to vanish, with vanishing derivative, for $d_{i k}=d_{\text {min }}$, where $0<d_{\text {min }}<d_{0}$ represents a minimum desired distance between the agents. The equations and shape of $c_{i k}$ is conceptually the same as of $b_{i j}$ in (7), so we refer again the reader to Fig. $2 \mathrm{~b}$

Notice that, because of (5) and the previous definitions, if agent $i$ gets too close to an obstacle or to another agent, the entire $i$-th row of $A$ will vanish, i.e., $A_{i j}=0, \forall j$. Agent $i$ would then become disconnected from the rest group, and any action aimed at preserving the connectivity would automatically enforce both obstacle and inter-agent collision avoidance.

Now consider the Laplacian matrix $L=\operatorname{diag}\left(\delta_{i}\right)-A$, $\delta_{i}=\sum_{j=1}^{N} A_{i j}$, associated to the graph $\mathcal{G}$. It is well known that a measure of the connectivity of $\mathcal{G}$ is given by $\lambda_{2} \geq 0$, the second smallest eigenvalue of $L: \lambda_{2}>0$ iff the graph is connected [16]. Since $A \in \mathcal{C}^{1}$, its eigenvalue $\lambda_{2}$ will be a differentiable function of $d_{i j}$ and $d_{i j o}$ for all the pair of agents, and will smoothly vanish as the graph approaches 
disconnection. It is then possible to build the potential function $V^{\lambda}$ described in Sect. II as a function of $\lambda_{2}$ and try to follow the gradient of this potential to keep $\lambda_{2}>0$ at all times.

Assume $\lambda_{2}^{\max }>\lambda_{2}^{\min }>0$ represent desired maximum and minimum values for $\lambda_{2}$. We define $V^{\lambda}\left(\lambda_{2}\right)$ as

$$
V^{\lambda}\left(\lambda_{2}\right)=\left\{\begin{array}{cc}
0 & \lambda_{2}<\lambda_{2}^{\min } \\
k_{\lambda} \tan ^{2}\left(\alpha_{\lambda} \lambda_{2}+\beta_{\lambda}\right) & \lambda_{2}^{\min }<\lambda_{2} \leq \lambda_{2}^{\max } \\
0 & \lambda_{2}>\lambda_{2}^{\max }
\end{array}\right.
$$

with $\alpha_{\lambda}=\frac{\pi}{2\left(\lambda_{2}^{\max }-\lambda_{2}^{\min }\right)}$ and $\beta_{\lambda}=-\alpha \lambda_{2}^{\max }$. This potential grows unbounded as $\lambda_{2} \rightarrow \lambda_{2}^{\text {min }}$ and vanishes (with vanishing derivative) for $\lambda_{2}=\lambda_{2}^{\max }$. Figure 2c shows an example for $\lambda_{2}^{\min }=0.1, \lambda_{2}^{\max }=1$ and $k_{\lambda}=10$.

We can compute the force generated on the agent $i$ by the potential $V^{\lambda}$ as $F_{i}^{\lambda}=\sum_{j \in \mathcal{N}_{i}}\left(\frac{\partial V^{\lambda}}{\partial x_{i j}}+\frac{\partial V^{\lambda}}{\partial x_{o_{i j}}}\right)$ which can be rewritten as $F_{i}^{\lambda}=\frac{\partial V^{\lambda}}{\partial \lambda_{2}} \sum_{j \in \mathcal{N}_{i}}\left(\frac{\partial \lambda_{2}}{\partial x_{i j}}+\frac{\partial \lambda_{2}}{\partial x_{o_{i j}}}\right)$. Evaluation of $\frac{\partial V^{\lambda}}{\partial \lambda_{2}}$ follows from the definition in 8 , while the terms in the sum can be computed as shown in [T6]:

$$
\sum_{j \in \mathcal{N}_{i}} \frac{\partial \lambda_{2}}{\partial x_{i j}}=\sum_{j \in \mathcal{N}_{i}} \frac{\partial A_{i j}}{\partial x_{i j}}\left(\nu_{2_{i}}-\nu_{2_{j}}\right)^{2}
$$

and similarly for the second term in the sum, with $\nu_{2}$ being the (normalized) eigenvector of $L$ associated to $\lambda_{2}$. From the definition of the weights in 677 , it follows that $\frac{\partial A_{i j}}{\partial x_{o_{i j}}}=$ $a_{i j} \frac{\partial b_{i j}}{\partial x_{o_{i j}}} c_{i} c_{j}$ and $\frac{\partial A_{i j}}{\partial x_{i j}}=\frac{\partial \bar{a}_{i j}}{\partial x_{i j}} b_{i j} c_{i} c_{j}+a_{i j} b_{i j} \frac{\partial\left(c_{i} c_{j}\right)}{\partial x_{i j}}$. The latter terms can be straightforwardly evaluated by taking the derivative of $a_{i j}$ and $c_{i} c_{j}$ w.r.t. $d_{i j}$ from the definition in 6-77, and by noting that $d_{i j}=\left\|x_{i}-x_{j}\right\|$ from which $\frac{\partial d_{i j}}{\partial x_{i j}}=\frac{x_{i j}}{\left\|x_{i j}\right\|}$ easily follows. On the other hand, evaluation of the term $\frac{\partial b_{i j}}{\partial x_{o_{i j}}}$ needs a formal definition of the line-of-sight/obstacle distance $d_{i j o}$ that was chosen as follows: let $s_{i j o}$ be the orthogonal projection of $o_{i j}$ onto the supporting line of $s_{i j}$ (the segment joining agents $i$ and $j$ ). If $s_{i j o}$ lies inside the segment boundaries, then $d_{i j o}$ is the standard point-line distance. If $s_{i j o}$ lies outside the segment boundaries and is, w.l.o.g., closer to the segment vertex $x_{i}$, then $d_{i j o}=\left\|x_{o_{i j}}\right\|$, and similarly in the symmetric case. Thus, $\frac{\partial b_{i j}}{\partial x_{o_{i j}}}$ can be evaluated by computing $\frac{\partial b_{i j}}{\partial d_{i j o}}$ from 77 and $\frac{\partial d_{i j o}}{\partial x_{o_{i j}}}$ depending on the particular case illustrated above.

We note that, apart from the (global) quantities $\lambda_{2}$ (needed in $\frac{\partial V^{\lambda}}{\partial \lambda_{2}}$ ) and $\nu_{2}$ (needed in 99 ), $F_{i}^{\lambda}$ could be evaluated locally since it only depends on the relative measurements $d_{i j}$, and $d_{i j o}, j \in \mathcal{N}_{i}$. Knowledge of $\lambda_{2}$ and $\nu_{2}$ could be obtained by a global observation of the group in order to recover the full Laplacian $L$. In our case, we chose to rely on the decentralized estimation strategy proposed in by Yang et al. in [16]. Therein, the authors show how each agent $i$ can obtain its own local estimation of $\lambda_{2}$, i.e., $\hat{\lambda}_{2}$, and of the $i$-th component of $\nu_{2}$, i.e., $\hat{\nu}_{2_{i}}$, by again exploiting only local and 1-hop information.We refer the reader to this work for all the details.

Therefore, by exploiting these results, an estimation $\hat{F}_{i}^{\lambda}$ of the true $F_{i}^{\lambda}$ can be implemented by every agent in a fully decentralized way. We would like to summarize the main features of the potential $V^{\lambda}$ and of $F_{i}^{\lambda}$ introduced so far:

1) although $V^{\lambda}$ is a global potential, reflecting global properties (connectivity) of the group, an estimation of $\hat{F}_{i}^{\lambda}$ can be computed in a fully decentralized way;

2) $V^{\lambda}$ will grow unbounded as $\lambda_{2} \rightarrow \lambda_{2}^{\min }>0$, thus enforcing connectivity of the group. Note that, during the motion, agents are fully allowed to break or create links as long as $\lambda_{2}>\lambda_{2}^{\mathrm{min}}$. This provides large amounts of flexibility to the group topology and geometry;

3) because of the shape of the terms $a_{i j}$ and $c_{i k}, k \in \mathcal{N}_{i}$, in 6 67, minimization of $V^{\lambda}$ will lead the agents $i$ and $j$ to keep a preferred inter-distance $d_{0}$, without getting too far or too close to each other, since in either cases their link would approach disconnection resulting in a decrease of $\lambda_{2}$. Similarly, because of the shape and definition of $b_{i j}$ in (7), each pair of neighbors will try to keep its line-of-sight not occluded by obstacles;

4) because of the term $c_{i} c_{j}$ in (5), $\lambda_{2} \rightarrow \lambda_{2}^{\min }$ as any inter-agent distance $d_{i j} \rightarrow d_{\text {min }}$. Indeed, as an agent $i$ approaches an agent $j$, the corresponding weight $c_{i j}$ will vanish, implying that $A_{i k} \rightarrow 0 \forall k \in \mathcal{N}_{i}$ (since the same weight $c_{i j}$ is present in all the $\left.A_{i k}, k \in \mathcal{N}_{i}\right)$. Agent $i$ will loose all its links with the other agents and become disconnected from the group. Therefore, minimization of $V^{\lambda}$ will prevent any inter-agent collision;

5) similarly to the previous case, because of the terms $b_{i j}$, any collision among agents and obstacles would imply $\lambda_{2} \rightarrow \lambda_{2}^{\mathrm{min}}$ and, therefore, an unbounded growth of $V^{\lambda}$. As an agent $i$ approaches an obstacle it will eventually become the closest point to the obstacle on all the links departing from it, i.e., on all the $s_{i k}, k \in \mathcal{N}_{i}$. At the minimum distance $d_{\min }^{o}$, all the $b_{i k}, k \in \mathcal{N}_{i}$, would vanish, leading to a disconnection of agent $i$ from the rest of group like in the previous case.

\section{B. Passivity of the slave side}

We refer to a split as the cancelation of the coupling forces between a pair of agents $i$ and $j$ (because of too large/little inter-distance or proximity to obstacles) and to a join as the (re-)establishment of the coupling, e.g., after a split. As shown in [2], a split does not threaten passivity of the slave side, while this, in general, is not the case for a join decision. When two agents join at some time $\bar{t}$, they instantaneously switch from a state characterized by no interaction to a new state where a spring-like coupling is (re-)created. In this situation, the potential used to model the inter-agent connection, say a generic $V$, could instantaneously jump from the value $V\left(\bar{t}^{-}\right)$ to a new value $V\left(\bar{t}^{+}\right)>V\left(\bar{t}^{-}\right)$depending on the particular state of the two agents, thus injecting extra energy $\Delta E=$ $V\left(\bar{t}^{+}\right)-V\left(\bar{t}^{-}\right)>0$ into the system.

Nevertheless in our case $\left(V=V^{\lambda}\right)$, no jumps are possible in any situation because of the way the weights $A_{i j}$ are designed. Indeed, when agents $i$ and $j$ split (or are initially disconnected) it is $A_{i j}=A_{j i}=0$, and the same condition holds when the two agents join again at some $\bar{t}$ : 
1) if $d_{i j o} \geq d_{\min }^{o}$ (line-of-sight not occluded) and $d_{i j}\left(\bar{t}^{-}\right)>D$ followed by $d_{i j}\left(\bar{t}^{+}\right)=D$ (a link is created), $A_{i j}\left(\bar{t}^{-}\right)=A_{i j}\left(\bar{t}^{+}\right)=0$ because of the weights $a_{i j}\left(d_{i j}\right)$;

2) if $d_{i j}<D$ (agents within the sensing range) and $d_{i j o}\left(\bar{t}^{-}\right)<d_{\min }^{o}$ followed by $d_{i j o}\left(\bar{t}^{+}\right)=d_{\min }^{o}$ (a link is created), $A_{i j}\left(\bar{t}^{-}\right)=A_{i j}\left(\bar{t}^{+}\right)=0$ because of the weights $b_{i j}\left(d_{i j o}\right)$.

Therefore, as $V^{\lambda}$ can never jump by construction, passivity of the slave side is guaranteed without the need of additional actions such as those reported in [2]. On the other side, nonpassive behaviors could happen due to estimation errors in evaluating $\hat{F}_{i}^{\lambda}$. We will now discuss how to still enforce passivity despite of this shortcoming.

1) Passive implementation of $\hat{F}_{i}^{\lambda}$ : we first introduce the fundamental concept of tanks [2, [12, 15]: the tanks are artificial energy storing elements that keep track of the energy dissipated by each agent because of the damping terms $B_{i}$. The energy stored in these reservoirs can be used without violating the passivity of the system. From Eq. (1), it follows that the energy dissipated by agent $i$ because of the damping is given by

$$
D_{i}=p_{i}^{T} M_{i}^{-T} B_{i} M_{i}^{-1} p_{i}
$$

Consider a tank with state $x_{t_{i}} \in \mathbb{R}$ and associated energy $T_{i}=$ $\frac{1}{2} x_{t_{i}}^{2}$ : we propose to adopt the following extended dynamics for the agents:

$$
\left\{\begin{array}{l}
\dot{p}_{i}=F_{i}^{\lambda}+F_{i}^{e}-w_{i} x_{t_{i}}-B_{i} M_{i}^{-1} p_{i} \\
\dot{x}_{t_{i}}=\alpha_{i} \frac{1}{x_{t_{i}}} D_{i}+w_{i}^{T} v_{i} \\
y_{i}=\left(v_{i}^{T} x_{t_{i}}\right)^{T}
\end{array} .\right.
$$

The total energy of the slave side is then now

$$
\mathcal{H}=\sum_{i=1}^{N}\left(\mathcal{K}_{i}+T_{i}\right)+V^{\lambda}
$$

and the Tank/Kinetic power flows become

$$
\left\{\begin{array}{l}
\dot{T}_{i}=\alpha_{i} D_{i}+x_{t_{i}} w_{i}^{T} v_{i} \\
\dot{\mathcal{K}}_{i}=\frac{\partial \mathcal{K}^{T}}{\partial p_{i}}\left(F_{i}^{\lambda}+F_{i}^{e}-B_{i} M_{i}^{-1} p_{i}\right)-v_{i}^{T} w_{i} x_{t_{i}}
\end{array} .\right.
$$

The input $w_{i} \in \mathbb{R}^{3}$ is meant to exchange energy between the tank $x_{t_{i}}$ and agent $i$ as can be seen from (13) while the quantity $\alpha_{i} \in\{0,1\}$ is a design parameter. If $\alpha_{i}=1$, all the energy dissipated because of the damping $B_{i}$ is stored back into the tank: such energy can be re-injected into the system without violating the passivity constraint. Because of the reasons reported in [6], it is wise to disable the storing of $D_{i}$ for avoiding an excess of energy stored that would allow to implement unstable behaviors in the system. Thus, $\alpha_{i}=1$ if $T_{i} \leq \bar{T}_{i}$ and $\alpha_{i}=0$ otherwise, where $\bar{T}_{i}$ is a suitable upper bound depending on the particular application. Furthermore, in order to avoid singularities in (11) (i.e. $x_{t_{i}}=0$ ), an additional threshold $\varepsilon>0$ is set restricting the amount of extractable energy from the tank.
As explained before, estimation errors in $\hat{F}_{i}^{\lambda}$ can potentially lead to a loss of passivity. To see why, consider the two energy storing elements in $3-4$, that is, the elastic potential $V^{\lambda}$ and the kinetic energy $\mathcal{K}=\sum_{i}^{N} \mathcal{K}_{i}$. Passivity is preserved if the energy exchange between these two elements occurs in a passive way, i.e., without the creation of extra energy excess. From (3) and previous definitions, the energy balance for the element $V^{\lambda}$ can be written as

$$
V^{\lambda}(t)-V^{\lambda}\left(t_{0}\right)=-\sum_{i=i}^{N}\left(\int_{t_{0}}^{t}\left(F_{i}^{\lambda}\right)^{T} v_{i} \mathrm{~d} t\right)+\int_{t_{0}}^{t} v_{o}^{T} F_{o} d t
$$

Since we are assuming that obstacles are passive, we have that $-\int_{t_{0}}^{t} v_{o}^{T} F_{o} d t \geq 0$, where we also assumed for simplicity that the initial energy stored in the obstacles is zero. Thus we have that

$$
V^{\lambda}(t)-V^{\lambda}\left(t_{0}\right) \leq-\sum_{i=i}^{N}\left(\int_{t_{0}}^{t}\left(F_{i}^{\lambda}\right)^{T} v_{i} \mathrm{~d} t\right)=\Lambda(t)
$$

Since $V^{\lambda}(t) \geq 0$, condition 15 implies that $\Lambda(t) \geq-V^{\lambda}\left(t_{0}\right)$, where $V^{\lambda}\left(t_{0}\right) \geq 0$, i.e., the well-known fact that total extractable energy from the (passive) element $V^{\lambda}$ is bounded by $V^{\lambda}\left(t_{0}\right)$. On the other hand, the energy absorbed by the kinetic energy element $\mathcal{K}$ (the slave side) is

$$
\Gamma(t)=\sum_{i=i}^{N}\left(\int_{t_{0}}^{t} v_{i}^{T} \hat{F}_{i}^{\lambda} \mathrm{d} t\right)
$$

In absence of estimation errors, $\Gamma(t)=-\Lambda(t)$, i.e., the interconnection of the two energy storing elements is powerpreserving $\Gamma(t)+\Lambda(t)=0$. On the other hand, estimation errors can potentially lead to $\Gamma(t)>-\Lambda(t)$, violating the passivity of the system. To overcome this obstacle, a possibility is to monitor the energy $\Gamma(t)$ absorbed by the slave side and ensure that $\Gamma(t) \leq-\Lambda(t) \leq V^{\lambda}\left(t_{0}\right), \forall t \geq t_{0}$.

To this end, we proceed as follows: first of all, since $V^{\lambda}\left(t_{0}\right)$ cannot be in general evaluated by the slave side (it would require exact knowledge of $\lambda_{2}$ ), we aim at the (more conservative) condition $\Gamma(t) \leq 0, \forall t \geq t_{0}$. Secondly, we note that $\Gamma(t)$ is a global quantity (it depends on the state of all the $N$ agents): therefore, by letting $\Gamma_{i}(t)=\int_{t_{0}}^{t} v_{i}^{T} \hat{F}_{i}^{\lambda} \mathrm{d} t$, we relax the condition $\Gamma(t)=\sum_{i=1}^{N} \Gamma_{i}(t) \leq 0$ to the (more conservative) $\Gamma_{i}(t) \leq 0$ that can be locally evaluated by every agent.

The passification strategy is then the following: while, during motion, $\Gamma_{i}(t) \leq 0$, agent $i$ can safely implement the force $\hat{F}_{i}^{\lambda}$ without extra actions. If $\Gamma_{i}(t)>0$, tank $T_{i}$ is exploited to compensate for the extra energy. In particular, the term $F_{i}^{\lambda}$ is set to 0 in Eq. $\sqrt{11}$ to avoid production of energy in the interconnection and the amount $\Gamma_{i}(t)>0$ is extracted from the tank allowing the agent to still implement $\hat{F}_{i}^{\lambda}$ while preserving the total energy balance. This is formally obtained by setting $w_{i}=-\beta_{i} \hat{F}_{i}^{\lambda} / x_{t_{i}}$ in (11) with $\beta_{i} \in\{0,1\}$ being a design parameter enabling/disabling the energy transfer from the tank to agent $i$. In particular, $\beta_{i}=1$ when $\Gamma_{i}>0$ and $\beta_{i}=0$ otherwise. Defining the matrices 


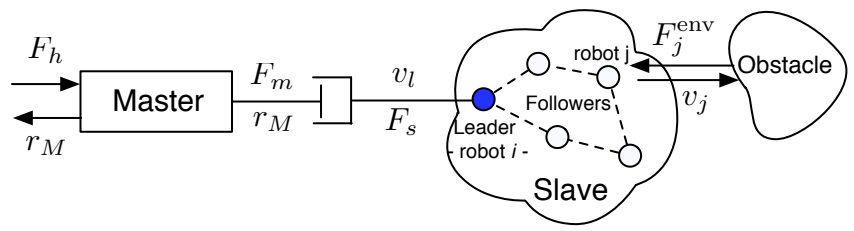

Fig. 4: The overall teleoperation system

$\Upsilon=\operatorname{diag}\left(-w_{i}\right), P=\operatorname{diag}\left(\frac{1}{x_{t_{i}}} p_{i}^{T} M_{i}^{-T}\right), \alpha=\operatorname{diag}\left(\alpha_{i}\right)$, and $\nabla \mathcal{H}=\left(\frac{\partial^{T} \mathcal{H}}{\partial p} \frac{\partial^{T} \mathcal{H}}{\partial x_{R}} \frac{\partial^{T} \mathcal{H}}{\partial x_{O}} \frac{\partial^{T} \mathcal{H}}{\partial x_{t}}\right)^{T}$ the slave side can be represented as

$\left\{\begin{array}{l}\left(\begin{array}{c}\dot{p} \\ \dot{x}_{R} \\ \dot{x}_{O} \\ \dot{x}_{t}\end{array}\right)=\left[\left(\begin{array}{cccc}0 & \mathcal{I} & -\mathbb{I} & \Upsilon \\ -\mathcal{I}^{T} & 0 & 0 & 0 \\ \mathbb{I}^{T} & 0 & 0 & 0 \\ -\Upsilon^{T} & 0 & 0 & 0\end{array}\right)-\left(\begin{array}{cccc}B & 0 & 0 & 0 \\ 0 & 0 & 0 & 0 \\ 0 & 0 & 0 & 0 \\ -\alpha P B & 0 & 0 & 0\end{array}\right)\right] \nabla \mathcal{H} \\ +G\left(\begin{array}{c}F^{\mathrm{e}} \\ v_{o}\end{array}\right) \\ \left(\begin{array}{c}v \\ F^{o}\end{array}\right)=G^{T} \nabla \mathcal{H}\end{array}\right.$

Proposition 1: The slave side is passive with respect to the storage function reported in Eq. (12).

Proof: Using Eq. (12), the following energy balance can be easily obtained:

$$
\dot{\mathcal{H}}=-\frac{\partial^{T} \mathcal{H}}{\partial p} B \frac{\partial \mathcal{H}}{\partial p}+\alpha \frac{\partial^{T} \mathcal{H}}{\partial x_{t}} P B \frac{\partial H}{\partial p}+v^{T} F^{\mathrm{e}}+v_{o}^{T} F^{o}
$$

Considering the definition of $P$, Eq. (18) becomes

$$
\begin{aligned}
\dot{\mathcal{H}}=-\frac{\partial^{T} \mathcal{H}}{\partial p} B \frac{\partial \mathcal{H}}{\partial p}+\alpha \frac{\partial^{T} \mathcal{H}}{\partial p} B \frac{\partial H}{\partial p} & +v^{T} F^{\mathrm{e}}+v_{o}^{T} F^{o} \leq \\
& \leq v^{T} F^{\mathrm{e}}+v_{o}^{T} F^{o}
\end{aligned}
$$

where the inequality comes from the fact that the energy stored in the tanks (the second term) is at most equal to the energy dissipated by the agents (the first term).

Tanks are continuously refilled by the energy dissipated by the agents and, therefore, it is very likely that the energy stored is sufficient for passively compensating the energy production due to the estimation error. This has been confirmed in our experiments. However, more energy could be left in the tanks by implementing less conservative (but more complex) strategies. For example, running a consensus on $\Gamma_{i}(t)$ can provide each agent with (an estimation of) the global quantity $\Gamma(t)$ : in this way, only the amount of energy strictly necessary for implementing the desired control action would be extracted by the tank, resulting in much less conservative bounds. Nevertheless, in case the energy stored in the tank falls below an emergency level, the damping of the corresponding agent can be raised to a maximum level. This has two advantages: first, more energy is dissipated and the tank is recharged more quickly and, second, the agent is forced to slow down and to eventually stop for avoiding collisions in case the tank is depleted and $\hat{F}_{i}^{\lambda}$ cannot be implemented.

\section{The Teleoperation SYSTEM}

The master can be a generic mechanical system and it can be modeled by the following Euler-Lagrange equations:

$$
M_{M}\left(x_{M}\right) \ddot{x}_{M}+C\left(x_{M}, \dot{x}_{M}\right) \dot{x}_{M}+D_{M} \dot{x}_{M}=F_{M}
$$

where $M_{M}$ represents the inertia matrix, $C\left(x_{M}, \dot{x}_{M}\right) \dot{x}_{M}$ is a term representing the centrifugal and Coriolis effects, $D_{M}$ is matrix representing both the viscous friction present in the system and any additional damping injection via local control actions. As often happens for master devices, we assume that gravity effects are compensated by a local controller. The variables $x_{M}$ and $\dot{x}_{M}$ represent the position and the velocity of the end-effector. A system described by Eq. 20] is passive with respect to the force-velocity pair $\left(F_{M}, v_{M}\right)$ [13], where $v_{M}:=\dot{x}_{M}$. This kind of passivity is well suited in standard passivity based bilateral teleoperation, where the velocity of the master and the velocity of the slave need to be synchronized.

Nevertheless, in our setting, in order to consider the difference between the workspace of the master and that of the robots at the slave side, we would like to transform the position of the master into a velocity setpoint for the leader at the slave side, and to feed back the mismatch between the master position and the actual leader velocity as a force cue at the master side in order to transmit to the user a feeling of the remote side. As reported in [2], it is possible to build a control loop that makes the master passive with respect to the pair $\left(F_{M}, r_{M}\right)$, where $r_{M}=\rho v_{M}+\sigma x_{M}$, where $\rho$ and $\sigma$ are strictly positive gains. By properly setting $\rho$ and $\sigma$ it is possible to make the contribution relative to the velocity negligible and to render the master passive with respect to a term that is arbitrarily close to the position and the force.

Exploiting the results developed so far, we have that both master and slave sides are passive systems. Thus, by designing a proper passive interconnection between the local and the remote systems will yield a passive bilateral teleoperation system characterized by a stable behavior in case of interaction with passive environments (as the obstacles, modeled as potentials, with which the group is interacting).

Suppose that agent $i$ is chosen as the leader. It is possible to write $F_{i}^{e}=F_{s}+F_{i}^{\text {env }}$, where $F_{i}^{\text {env }}$ is the component of the force due to the interaction with the external environment and $F_{s}$ is the component due to the interaction with the master side. Similarly, we can decompose $F_{M}$ as $F_{M}=F_{m}+F_{h}$, where $F_{h}$ is the component due to the interaction with the user and $F_{m}$ is the force acting on the master because of the interaction with the slave.

For achieving the desired teleoperation behavior, we propose to join master and slave using the following interconnection:

$$
\left\{\begin{array}{l}
F_{s}=b\left(r_{M}-v_{i}\right) \\
F_{m}=-b\left(r_{M}-v_{i}\right)
\end{array}\right.
$$

This is equivalent to joining the master and the leader using a damper which generates a force proportional to the difference of the two velocity-like variables of the master and the leader. Since $r_{M}$ is "almost" the position of the master, we have that the force fed back to the master and the control action sent to the leader are the desired ones. The overall teleoperation system is represented in Fig. 4 and it consists of the interconnection of a passive master side, a passive interconnection and a passive slave side. Recalling that the 


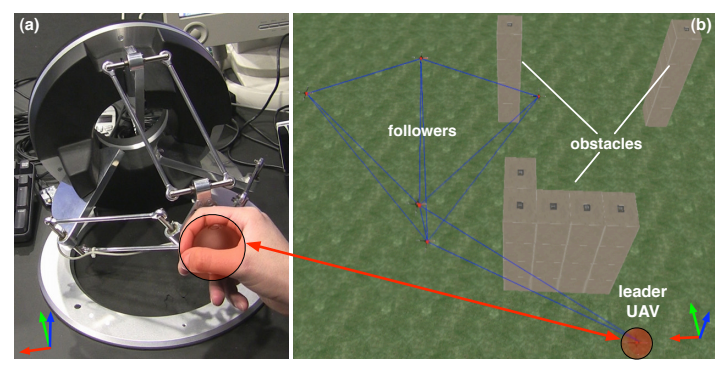

Fig. 5: Human/Hardware in the loop setup.

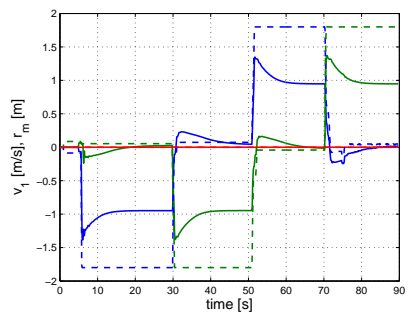

(a)

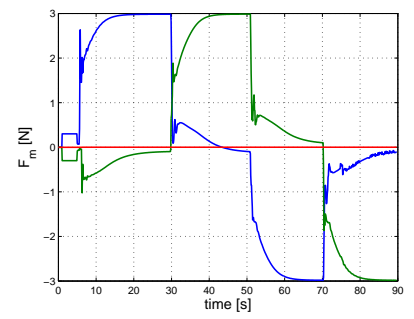

(b)
Fig. 6: Results of the first HHIL simulation. Left: superimposition of the leader velocity $v_{1}(t)$ and the master command $r_{m}(t)$. Right: the force $F_{m}(t)$ displayed to the human operator on the master side

interconnection of passive systems is again passive [13], we have that the teleoperation system is passive, as desired ${ }^{4}$

\section{Human/HaRdware-IN-THE-LOOP Simulations}

To validate the theoretical framework developed so far we conducted several HHIL simulations (see Fig. 5) with a commercial haptic device, the Omega.3.5 as a master robot. The Omega. 3 is a 3 -DOF haptic device with 3 translational actuated axes, and its local control loop runs at about $2.5 \mathrm{kHz}$ on a dedicated gnu/linux machine. The UAVs dynamics and control logic, on the other hand, were simulated in a custommade simulation environment based on the Ogre3D engine (for 3D rendering and computational geometry computations), the PhysX libraries for simulating the physical interaction between the UAVs and the environment, and the MIP framework for the multi-robot communication and control aspects ${ }^{6}$

In the following, we assume w.l.o.g. that the leader is agent 1 over a total of $N=6$ agents composing the group. During the first simulation, we tested the behavior of the teleoperation system during free motion, i.e., sufficiently away from obstacles and in quasi steady-state conditions. The goal was to show the stability of the teleoperation system. Figure $6 \mathrm{a}$ shows a superimposition of the leader velocity $v_{1}$ (solid lines) and the master variable $r_{M}$ (dashed lines). We can see that the leader velocity tracks the master command with a constant

\footnotetext{
${ }^{4}$ If needed, one can also passively consider the communication delay between local and remote site using one of the techniques developed for single master single slave telemanipulation systems, as, e.g., wave variables [9]. In this way, the system would keep on exhibiting a stable behavior independently of any delay between local and remote site.

5 http://www.forcedimension.com

6 http://www.ogre3d.org http://www.nvidia.com/object/physx_new.html http://www.dis.uniroma1.it/ labrob/software/MIP
}

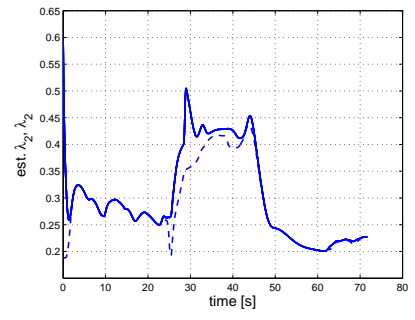

(a)

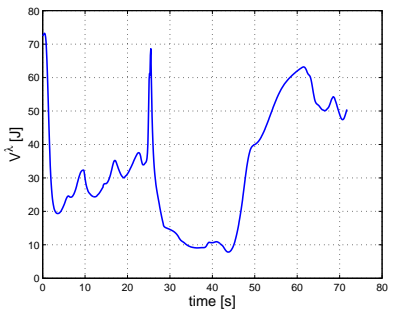

(b)
Fig. 7: Results of the second HHIL simulation. Left: superimposition of $\hat{\lambda}_{2}^{i}(t), i \in 1 \ldots N$ (solid lines), and $\lambda_{2}(t)$ (dashed line). Note how the internal estimations $\hat{\lambda}_{2}^{i}(t)$ are almost perfectly coincident in a unique solid line. Right: behavior of $V^{\lambda}(t)$ which never diverges to infinity as $\lambda_{2}(t)>\lambda_{2}^{\min }=0.1, \forall t \geq t_{0}$

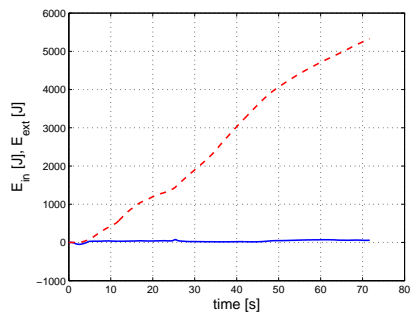

(a)

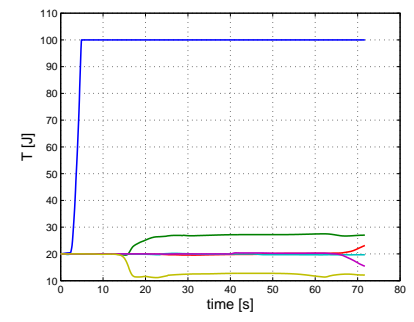

(b)
Fig. 8: Results of the second HHIL simulation. Left: behavior of $E_{\text {in }}(t)$ (blue solid line) and $E_{\text {out }}(t)$ (red dashed line) showing that the passivity condition (19) is always met. Right: Behavior of the tank energies $T_{i}(t), \forall t \geq t_{0}$. The tanks never deplete, allowing the passive implementation of $\hat{F}_{i}^{\lambda}(t)$

steady-state error: in particular, at every change in the (piecewise constant) $r_{M}, v_{1}$ responds with an initial rise and then settles down to the steady-state value. This behavior is the normal outcome of our teleoperation framework and is due to the viscous resistance that the followers exert on the leader because of their damping terms $B_{i}$. After the initial transient, the leader starts pulling all the followers and is constantly dragged by them. Figure 6b shows the force $F_{m}$ displayed to the human operator. We can note that the force behavior correctly reproduces the effects of the followers on the leader: the operator is provided with a force opposing the commanded motion, thus conveying the information that he is actually pulling a 'load with friction'.

In the second experiment, we tested the teleoperation scheme in a more general motion involving several split and rejoin maneuvers in a environment cluttered with obstacles. Figure $7 \mathrm{a}$ shows the behavior of $\hat{\lambda}_{2}^{i}(t), i \in 1 \ldots N$ (solid lines), and of $\lambda_{2}(t)$ (dashed line). The quantities $\hat{\lambda}_{2}^{i}(t)$ are the internal estimations of $\lambda_{2}(t)$ for each agent $i$ and are almost perfectly coincident: the 'single' solid line in the plot is actually a superimposition of the $N \hat{\lambda}_{2}^{i}(t)$, showing that all the agents agree on the current graph connectivity. One can also appreciate the transitory mismatches between $\hat{\lambda}_{2}^{i}(t)$ and $\lambda_{2}(t)$ due to the estimation convergence. However, despite the estimation errors, the agents keep being connected (i.e., within the sensing range $D$ and without suffering from obstacle or 
inter-agent collisions) as $\lambda_{2}(t)>\lambda_{2}^{\min }=0.1, \forall t \geq t_{0}$. This is also confirmed by the behavior of $V^{\lambda}(t)$ shown in Fig. $7 \mathrm{~b}$ which does not diverge to infinity over time.

Finally, Figs $8 \mathrm{a}-8 \mathrm{~b}$ are meant to illustrate that passivity of the slave-side is enforced during the motion. Figure $8 \mathrm{a}$ shows the behavior of $E_{\text {in }}(t)=\mathcal{H}(t)-\mathcal{H}\left(t_{0}\right)$ (blue solid line) and $E_{\text {ext }}(t)=\int_{t_{0}}^{t}\left(v^{T} F^{\mathrm{e}}+v_{o}^{T} F^{o}\right) \mathrm{d} t$ (red dashed line), i.e., the integral version of the passivity condition (19). The plots confirm that the inequality in (19) is always met and, thus, that the slave-side behaves in a passive way w.r.t. the external forces. Figure $8 \mathrm{~b}$, on the other hand, shows the behavior of $T_{i}(t), i \in 1 \ldots N$ (the tank energies of the $N$ agents). The blue line, rising to $\bar{T}=100[\mathrm{~J}]$ at about $t=3[\mathrm{~s}]$ is $T_{1}(t)$, i.e., the tank energy of the leader. Since the leader is (almost) always pulling the followers, i.e., it is releasing energy into the elastic potential $V^{\lambda}$, its $\Gamma_{1}(t)$ stays negative and its tank $T_{1}(t)$ is not exploited by the passive strategy illustrated in Sect. II-B1 $T_{1}(t)$ keeps on storing the dissipated energy $D_{1}(t)$ until it reaches the maximum level $\bar{T}$. The followers, on the other hand, receive energy from the elastic potential $V^{\lambda}$ so that $\Gamma_{i}(t)>0, i \in 2 \ldots N$ : the tanks $T_{i}(t)$ are then exploited to implement $\hat{F}_{i}^{\lambda}$. This is evident by the fact that all the tank levels except $T_{1}(t)$ do not increase over time but, on average, the energy stored from $D_{i}(t)$ is reused to implement $\hat{F}_{i}^{\lambda}$ in a passive way. Nevertheless, no tank depletes confirming that the conservative strategy of Sect. II-B1] is not too restrictive for the agent motion.

A videoclip showing a representative experiment is downloadable from http://dl.dropbox.com/u/18694382/video1.mov

\section{COnClusions And Future Work}

In this paper we have shown how to design a passivity based control strategy to allow the bilateral teleoperation of a group of UAVs by means of a single remote human operator while enforcing global connectivity of the group in a decentralized way. To this end, we proposed a unique connectivity potential function $V^{\lambda}$ that encodes sensing and visibility (line-of-sight) constraints, as well as inter-agent and obstacle avoidance. We have shown that every agent can implement the gradient of this potential in a decentralized way while preserving the passivity of the overall slave-side system. This is a crucial point in order to embed our multi-agent slave side within a bilateral teleoperation scheme, where a human-controlled master device gives motion commands to the slave and receives a suitable force feedback from the remote (UAV) side. The approach was validated by means of HHIL simulations.

We are currently investigating the possibility of having more leaders at the slave side in order to have a better control of the motion of the UAVs. Finally, we also plan to passively implement some extra forces at the master side in order to convey some extra information about the connectivity and for improving the telepresence feeling of the user.

\section{ACKNOWLEDGEMENTS}

This research was partly supported by WCU (World Class University) program funded by the Ministry of Education, Science and Technology through the National Research Foundation of Korea (R31-10008).

\section{REFERENCES}

[1] Y. Cao and W. Ren. Distributed coordinated tracking via a variable structure approach - part I: consensus tracking. part II: swarm tracking. In American Control Conference, pages 4744-4755, 2010.

[2] A. Franchi, P. Robuffo Giordano, C. Secchi, H. I. Son, and H. H. Bülthoff. A passivity-based decentralized approach for the bilateral teleoperation of a group of UAVs with switching topology. In 2011 IEEE Int. Conf. on Robotics and Automation, pages 898-905, Shanghai, China, May 2011.

[3] N. Guenard, T. Hamel, and L. Eck. Control Laws For The Tele Operation Of An Unmanned Aerial Vehicle Known As An X4flyer. In 2006 IEEE/RSJ Int. Conf. on Intelligent Robots and Systems, pages 3249-3254, 2006.

[4] B. Hannaford. Stability and performance tradeoffs in bi-lateral telemanipulation. In 1989 IEEE Int. Conf. on Robotics and Automation, pages 1764-1767, Scottsdale, AZ, May 1989.

[5] M. Ji and M. Egerstedt. Distributed coordination control of multiagent systems while preserving connectedness. IEEE Trans. on Robotics, 2007.

[6] D. Lee and K. Huang. Passive-set-position-modulation framework for interactive robotic systems. IEEE Trans. on Robotics, 26(2):354 -369, april 2010. ISSN 1552-3098.

[7] N. E. Leonard and E. Fiorelli. Virtual leaders, artificial potentials and coordinated control of groups. In 40th IEEE Conf. on Decision and Control, pages 2968-2973, Orlando, FL, Dec. 2001.

[8] R. M. Murray. Recent research in cooperative control of multi-vehicle systems. ASME Journal of Dynamic Systems, Measurement and Control, 129:571-583, 2007.

[9] G. Niemeyer and J.-J Slotine. Telemanipulation with time delays. International Journal of Robotics Research, 23(9):873890, 2004

[10] G. Notarstefano, K. Savla, F. Bullo, and A. Jadbabaie. Maintaining limited-range connectivity among second-order agents. In American Control Conference, pages 2134-2129, 2006.

[11] E. J. Rodriguez-Seda, J. J. Troy, C. A. Erignac, P. Murray, D. M. Stipanovic, and M. W. Spong. Bilateral teleoperation of multiple mobile agents: Coordinated motion and collision avoidance. IEEE Trans. on Control Systems Technology, 18(4): 984-992, 2009. ISSN 1063-6536.

[12] C. Secchi, S. Stramigioli, and C. Fantuzzi. Position drift compensation in port-hamiltonian based telemanipulation. In 2006 IEEE/RSJ Int. Conf. on Intelligent Robots and Systems, Beijing, China, 2006.

[13] C. Secchi, S. Stramigioli, and C. Fantuzzi. Control of Interactive Robotic Interfaces: a port-Hamiltonian Approach. Springer Tracts in Advanced Robotics. Springer, 2007.

[14] S. Sirouspour. Modeling and control of cooperative teleoperation systems. IEEE Trans. on Robotics, 21(6):1220-1225, 2005.

[15] S. Stramigioli, R. Mahony, and P. Corke. A novel approach to haptic tele-operation of aerial robot vehicles. In 2010 IEEE Int. Conf. on Robotics and Automation, pages 5302-5308, May 2010.

[16] P. Yang, R. A. Freeman, G. J. Gordon, K. M. Lynch, S. S. Srinivasa, and R. Sukthankar. Decentralized estimation and control of graph connectivity for mobile sensor networks. Automatica, 46:390-396, 2010.

[17] M. M. Zavlanos and G. J. Pappas. Potential fields for maintaining connectivity of mobile networks. IEEE Trans. on Robotics, 23(4):812-816, 2007. 